\title{
A New Method to Identify Reservoirs in Tight Sandstones Based on the New Model of Transverse Relaxation Time and Relative Permeability
}

\author{
Yuhang Guo, Baozhi Pan, and Lihua Zhang \\ College of Geo-Exploration Science and Technology, Jilin University, Changchun, China \\ Correspondence should be addressed to Yuhang Guo; 124563749@qq.com
}

Received 14 January 2017; Accepted 9 March 2017; Published 19 April 2017

Academic Editor: Xiaorong Luo

Copyright (C) 2017 Yuhang Guo et al. This is an open access article distributed under the Creative Commons Attribution License, which permits unrestricted use, distribution, and reproduction in any medium, provided the original work is properly cited.

Relative permeability and transverse relaxation time are both important physical parameters of rock physics. In this paper, a new transformation model between the transverse relaxation time and the wetting phase's relative permeability is established. The data shows that the cores in the northwest of China have continuous fractal dimension characteristics, and great differences existed in the different pore size scales. Therefore, a piece-wise method is used to calculate the fractal dimension in our transformation model. The transformation results are found to be quite consistent with the relative permeability curve of the laboratory measurements. Based on this new model, we put forward a new method to identify reservoir in tight sandstone reservoir. We focus on the Well M in the northwestern China. Nuclear magnetic resonance (NMR) logging is used to obtain the point-by-point relative permeability curve. In addition, we identify the gas and water layers based on new $T_{2}-K_{r}$ model and the results showed our new method is feasible. In the case of the price of crude oil being low, this method can save time and reduce the cost.

\section{Introduction}

The phenomenon of Nuclear Magnetic Resonance (NMR) was discovered in 1946, and since then NMR has been widely used in various fields. NMR logging technology has been rapidly developed in recent years, with confirmed favorable results in the evaluation of reservoir permeability. NMR logging technology has a unique fluid sensitivity and is able to provide information regarding the fluid number of rock pores, fluid properties, and fluid-bearing pore sizes. Since this data cannot be obtained by other conventional logging methods, it thereby provides very important information for reservoir descriptions and evaluations [1]. With regard to NMR logging, a spin echo string is collected to obtain stratum information, of which the distribution of the retrieved $T_{2}$ (transverse relaxation time) is closely related to the pore size and can be expressed as the porosity distribution through calibration. The movable fluid (MFFI), unmovable fluid (BVI), and other information can also be obtained from the $\mathrm{T}_{2}$ distribution. Through the core experimental analysis of the target area, accurate $T_{2}$ cutoff values can be acquired to determine the irreducible water saturation measurements.

The relative permeability refers to the ratio between the effective permeability of each fluid type and the absolute permeability of the rock itself, in the cases of saturated multiphase fluid in the rock pores. The relative permeability is related to the rock's wettability and fluid saturation, and the relative permeability curve can be obtained by laboratory measurements. Purcell [2] proposed a model based on the capillary pressure curves. Burdine (1953) proposed similar equations in which the tortuosity ratio is introduced in the model. Brooks and Corey [3] made further research on calculating relative permeability based on capillary pressure. The model modified by Brooks and Corey has been widely used in many studies. Feng et al. (2016) proposed a new relative permeability model in which the pore size distribution, the tortuosity, and the gas-water spatial distribution were all considered. Combined with a capillary pressure curve, the shift of the transverse relaxation time $\left(T_{2}\right)$ distribution of Nuclear Magnetic Resonance is used to determine the parameters in 
the model. But the model has too many variables, which is hard to apply in practical logging evaluation.

In recent years, more and more researches focused on using fractal theory to establish the relationship between the relative permeability and other parameters. The fractal theory, which was developed in the 1970 s, is a rational tool used to describe complex and irregular phenomena and processes and provides a good method for research regarding the microscopic pore structure of porous media [4]. The fractal theory has been applied in the oil industry with fruitful results. A molecular adsorption method was applied by Pfeifer and Avnir [5] to obtain the pores of the reservoir rocks with fractal structure. On the basis of the fractal theory, the transformation relationship between the capillary pressure, relative permeability, and resistivity index was established by Li (2011). Then, Li's transformation model was improved by Pairoys (2013), and favorable sample effects have been achieved in particular regions. The transformation between $T_{2}$ and the resistivity index was discussed by Ge et al. [6], based on the fractal theory and the linear relationship between $P_{C}$ (the capillary pressure) and $T_{2}$. In accordance with the study results put forward by $\mathrm{Li}$, the relationship between the permeability, $T_{2}$, and capillary pressure was used to achieve the transformation relationship between the relative permeability and $T_{2}$ by Bai et al. [7]. However, it can be seen from the eventual transformation results that the residual water and oil saturation were not considered and have a certain deviation from experimental measurement results. In addition, in practical applications, such as oil shale, samples are difficult to obtain. Also, the experiment takes longer time, and it is impossible to measure all of the samples in well. A solution is necessary for finding a way to obtain the relative permeability curve with depth.

The novelty of this paper is using a combination of the previous research results regarding the links between capillary pressure, resistivity index, relative permeability, and transverse relaxation time; the transformation relationship between the transverse relaxation time and the relative permeability is derived. All experimental cores are taken from tight sandstone reservoir in northwest China. Based on the rock resistivity experiment experimental data and the mercury injection test data, the parameter expression in the transformation relationship is given in order to discuss the control factors and their influences. It is obvious that the transformation results are better than those of the known methods, which thereby provided a theoretical basis for the relative permeability curve with changes over depth through the NMR logging data. The model is applied in the northwest of China to obtain the relative permeability curve with depth, as well as identify the gas and water yield layers. It is a new and reliable reservoir identification method.

\section{Theoretical Background}

2.1. Relationship between the Capillary Pressure $\left(P_{C}\right)$ and the Resistivity Index (I). In the Archie equation, the relationship between the resistivity index and water saturation is shown as follows:

$$
I=\frac{b}{S_{w}^{n}}=\frac{R_{t}}{R_{0}} .
$$

$I$ is the resistivity index; $b$ is the constant of the Archie equation; $n$ is the saturation index; $R_{t}$ is the sample resistivity; $R_{0}$ is the rock resistivity in the case of $100 \%$ water bearing; and $S_{w}$ is the water saturation. In this study, the $I-S_{w}$ curves are a single fractal dimension, and $b$ and $n$ are the fixed values throughout the saturation distribution.

The capillary pressure can be expressed as follows:

$$
P_{C}=P_{n w}-P_{w}=\frac{2 \sigma \cos \theta}{r},
$$

where $P_{n w}$ and $P_{w}$ are the pressures of the nonwetting phase and wetting phase fluids, respectively; $P_{C}$ is the capillary pressure; $r$ is the capillary radius; $\sigma$ is the tension of the twophase fluid boundary surface; and $\theta$ is the wetting angle. It is clear that the capillary pressure is inversely proportional to the capillary radius. The relationship between the capillary pressure and the saturation can be established through a mercury injection experiment.

Toledo et al. [8] considered $P_{C}$ is a function of wetting phase saturation and fractal dimension.

$$
S_{W} \propto\left(P_{C}\right)^{-\left(3-D_{f}\right)},
$$

where $D_{f}$ is the fractal dimension. Based on the fractal model of the porous media, the relationship between the capillary pressure and the resistivity index was given by Li [9] .

$$
P_{C D}=I^{\beta}=\frac{P_{C}}{P_{e}} \text {. }
$$

Among these, $\beta$ is the index related to the water-film thickness, which is the function of the fractal function $D_{f}$; $P_{C D}$ is the dimensionless capillary pressure; and $P_{e}$ is the entry capillary pressure.

2.2. Relationship between the Capillary Pressure $\left(P_{C}\right)$ and the Nuclear Magnetic Transverse Relaxation Time $\left(T_{2}\right)$. The relationship between $P_{C}$ and $T_{2}$ is relatively well constrained due the relationship to the pore structure. The methods of using the $T_{2}$ distribution for transforming the pseudo capillary pressure curve mainly include a linear method and a power function method.

In the linear method [10], it is assumed that a linear relationship exists between $P_{C}$ and the reciprocal of $T_{2}$ as detailed below:

$$
P_{C}=\frac{2 \sigma \cos \theta}{r}=\frac{C_{1}}{T_{2}} .
$$

In the equation, $C_{1}$ is the linear transformation coefficient and can be obtained by the core data analysis. The power function method is more frequently applied, and the relationship between $T_{2}$ and the corresponding capillary radius has been given by He et al. [11, 12] as follows:

$$
T_{2}=m_{t} r^{n_{t}},
$$


where $m_{t}$ and $n_{t}$ are the empirical coefficients, obtained according to the core data analysis. The power function method is suitable for low-permeability reservoirs containing complex pore structure. Therefore, the power function relationship has been used in this study.

2.3. Relationship between the Relative Permeability $(\mathrm{Kr})$ and the Resistivity Index (I). Based on Darcy's law and Ohm's law, Li [14] proposed a model to describe the relationship between the wetting phase relative permeability and the resistivity index:

$$
k_{r w}=S_{W}^{*} \frac{1}{I}
$$

where $k_{r w}$ is the relative permeability of the wetting phase. $I$ is the resistivity index. $S_{W}^{*}$ is effective saturation which can be written:

$$
S_{W}^{*}=\frac{S_{w}-S_{w i r}}{1-S_{w i r}}
$$

where $S_{w}$ is the wetting phase saturation and $S_{w i}$ is the irreducible wetting phase saturation of the wetting phase. Following Li's research, Pairoys introduced the residual nonwetting phase saturation into his model. At the same time, $I^{*}$, the normalized or effective resistivity index, and $I_{\min }$, the minimum resistivity index obtained at residual nonwetting phase saturation $S_{n w r}$, were proposed.

$$
\begin{aligned}
k_{r w} & =S_{W}^{*} \frac{1}{I^{*}}, \\
S_{W}^{*} & =\frac{S_{w}-S_{w i r}}{1-S_{w i r}-S_{n w r}} .
\end{aligned}
$$

\section{New $T_{2}-K_{r}$ Model and Validation}

3.1. Wetting Phase Relative Permeability Calculated by the New Model. Although the relationships between $T_{2}$ and $P_{C}$, as well as between $P_{C}$ and $I$, have been extensively studied [10-14], research on the relationship between $T_{2}$ and $I$ must be established and requires further development. By combining the previous research on the relationship between the capillary pressure and the resistivity index, the relationship between $T_{2}$ and $I$ was studied incorporating the fractal theory $[6,7]$ (Zhang et al., 2015). The pore volume distribution expression of the known space is shown as follows:

$$
V \propto r^{3-D_{f}}
$$

In the equation, $V$ is the volume of the pores with the radius of $r$ among rock samples; and this equation is used to derive $r$.

$$
\frac{d V}{d r} \propto r^{2-D_{f}}
$$

In accordance with the principle of fractal geometry, the integral was carried out on the above equation in order to obtain the expression of the cumulative pore volume $V_{r}$ with a pore size less than $r$.

$$
V_{r}=\int_{r_{\min }}^{r} a_{v} r^{2-D_{f}} d r=b_{v}\left(r^{3-D_{f}}-r_{\min }^{3-D_{f}}\right) .
$$

In the equation, $r_{\min }$ is the minimum pore radius of the reservoir rocks; and $a_{v}$ is the proportionality constant $b_{v}=$ $a_{v} /\left(3-D_{f}\right)$. The total pore volume $V$ of the reservoir is as follows:

$$
V=b\left(r_{\max }^{3-D_{f}}-r_{\min }^{3-D_{f}}\right)
$$

Through (12) and (13), the expression of the cumulative volume fraction $S_{r}$ of the pore with a size less than $r$ can be obtained.

$$
S_{r}=\frac{V_{r}}{V}=\frac{r^{3-D_{f}}-r_{\min }^{3-D_{f}}}{r_{\max }^{3-D_{f}}-r_{\min }^{3-D_{f}}}
$$

Due to $r_{\min } \ll r_{\max }$ and $r_{\min } \ll r$, (14) can be simplified as follows:

$$
S_{r}=\left(\frac{r}{r_{\max }}\right)^{3-D_{f}}
$$

In reservoir physics, $S_{r}$ is the wetting phase saturation $S_{w}$. Equation (2) is substituted into (15) to obtain the following:

$$
S_{w}=S_{r}=\left(\frac{P_{c}}{P_{c \min }}\right)^{D_{f}-3}=P_{C D}^{D_{f}-3} .
$$

$P_{C D}$ is dimensionless, for the purpose of eliminating the influence of the fluid, rather than the fluid correction. In regard to the complex pore structure, the power function method was used to substitute (6) into (15), and the following could be obtained:

$$
S_{w}=S_{r}=\left(\frac{T_{2}}{T_{2 \max }}\right)^{\left(3-D_{f}\right) / n_{t}}=\left(T_{2 D}\right)^{\left(3-D_{f}\right) / n_{t}},
$$

where $T_{2 \max }$ is the maximum transverse relaxation time, corresponding to $r_{\max }$, and $T_{2 D}$ is the dimensionless transverse relaxation time $T_{2 D}=T_{2} / T_{2 \max }$. Therefore, the relationship between the $T_{2}$ distribution and the wetting phase saturation could be obtained. Equation (1) is substituted into (17), and the following equation was easily obtained:

$$
T_{2 D}=b_{t}(I)^{-\alpha} \text {, }
$$

where $\alpha$ is the index related to the pore radius distribution and relaxation time index and $b_{t}$ is the transformation factor, which can be obtained from the rock experiment data.

$$
\begin{aligned}
& \alpha=\frac{n_{t}}{n\left(3-D_{f}\right)}=n_{t} \beta, \\
& b_{t}=b^{n_{t} / n\left(3-D_{f}\right)}=b^{\alpha} .
\end{aligned}
$$

Based on the fractal theory and the relationship between the transverse relaxation time $T_{2}$, capillary pressure $P_{C}$, 
resistivity index $I$, and relative permeability $K_{r}$, the transformation relationship between the water-phase relative permeability and the $T_{2 D}$ dimensionless transverse relaxation time was obtained, including the parameters $\alpha, n$, and $b$. These parameters could be obtained from the experimental data. Combining (7) and (17)-(19), the $k_{r w}-T_{2}$ model can be derived as the following equation:

$$
K_{r w}=\frac{T_{2 D}^{1 / \alpha}}{b}\left(\frac{T_{2 D}^{1 / \alpha n}-S_{i r}}{1-S_{i r}}\right) .
$$

The parameters in (20) are all derived from the above equation. It is worth noting that $b$ is equal to 1.0084 which is showed in Figure 2 (data from experiment). In general, $b$ is a constant $(b=1)$. The nonwetting phase's relative permeability was obtained by (21). This equation was derived from a Brooks-Corey model, which was applied by Li [14] to more effectively solve the nonwetting phase's relative permeability.

$$
K_{r n w}=\left(1-S_{w}^{*}\right)^{2}\left(1-\left(S_{w}^{*}\right)^{\lambda /(2+\lambda)}\right)
$$

In (20), $S_{i r}$ is the residual water saturation. In many of the previous research results, the solution of $S_{w}^{*}$ takes into account the residual gas saturation $S_{o r} . \lambda$ is the pore size distribution index and can be calculated from capillary pressure data [3]. Therefore, this model was also correspondingly changed into (22). However, it was found that, in the actual application, better effects could be achieved without consideration of the residual gas saturation.

$$
K_{r w}=\frac{T_{2 D}^{1 / \alpha}}{b}\left(\frac{T_{2 D}^{1 / \alpha n}-S_{i r}}{1-S_{i r}-S_{o r}}\right) .
$$

It should be noted that the model proposed by Li [9] and Ma et al. [15] was based on Darcy's law, Ohm's law, and Poiseuille's law and expounded the relationship between the relative permeability and the resistivity index. In the derivation process of the model of Ma et al. [15], (23) was obtained, of which $L_{a}$ and $L_{w}$ were equivalent path lengths under the saturated and unsaturated water conditions, respectively. In this model, the pore radius is assumed as the cylinder, and $r_{a}$ and $r_{w}$ represent the equivalent pore radius under corresponding status, respectively. In (23), $r_{a}^{4}$ is equivalent to the increase of the weight of the pore radius under saturated water conditions, which indicates that the relative permeability is strongly influenced by the pore radius, while the transverse relaxation time is the physical variable closely related to the pore radius. In combination with previous research views, it was also proven that the application of the nuclear magnetic $T_{2}$ distribution for the purpose of establishing a new model of the relative permeability was reliable. Meanwhile, the related parameters in (20) characterized the internal relationship among the resistivity index, relative permeability, and transverse relaxation time.

$$
K_{r w}=\frac{L_{a}}{L_{w}} \frac{\pi r_{w}^{4}}{\pi r_{a}^{4}}
$$

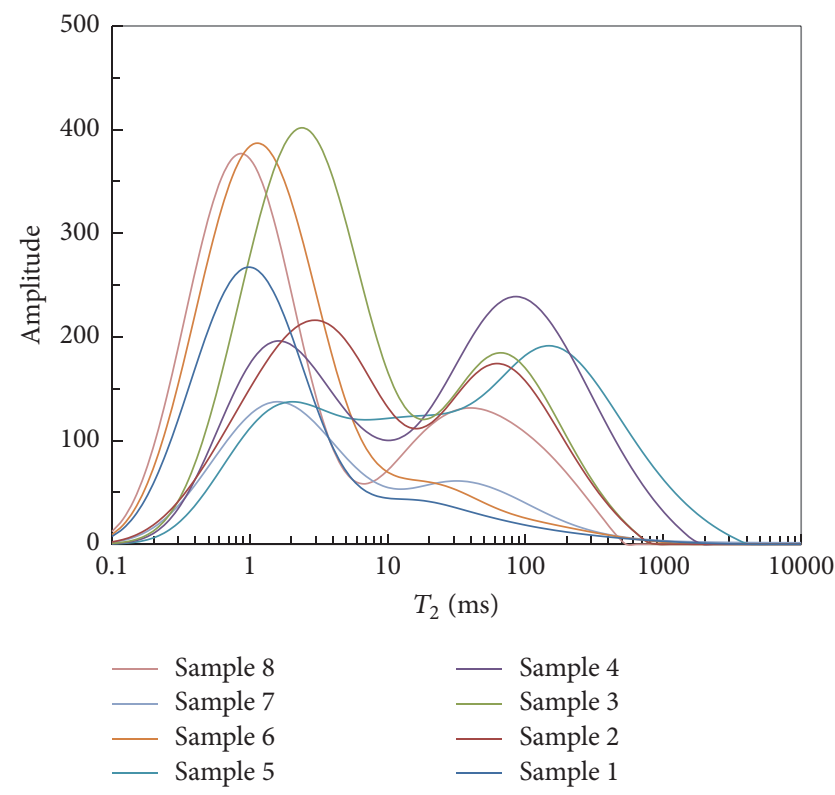

FIGURE 1: $T_{2}$ distribution map of the 8 samples.

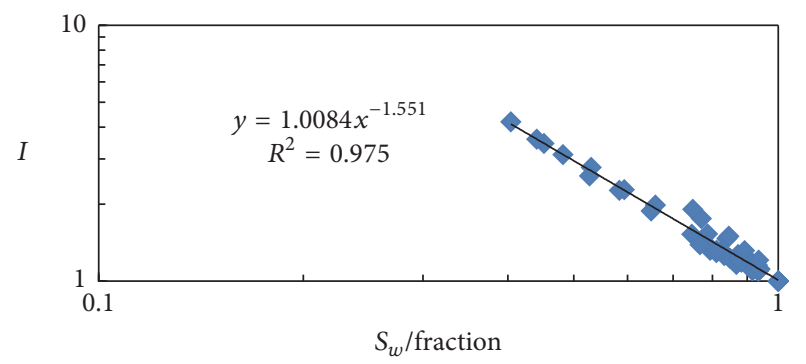

FIGURE 2: Relationship between the resistivity index and the water saturation of the 8 samples.

3.2. Results and Discussion. Tight sandstone reservoir samples were selected for the comprehensive laboratory measurements, in order to obtain the capillary pressure curve, relative permeability curve, core nuclear magnetic $T_{2}$ distribution, and resistivity index curve. The capillary pressure and the resistivity index were measured in RCS760 equipment produced by the Coretest company at the same condition (temperature: $55^{\circ} \mathrm{C}$, pore pressure: $14 \mathrm{Mpa}$, pressure of surrounding rock: $30 \mathrm{Mpa}$, and frequency: $1 \mathrm{KHz}$ ). The NMR experiments were performed in the modified MARAN II equipment operating at a Larmor frequency of $4 \mathrm{MHz}$ at ambient pressure and $55^{\circ} \mathrm{C}$. By using a CPMG pulse sequence, transverse relaxation time could be obtained [1]. The brine used for experiment had a salinity of $30000 \mathrm{ppm}$ with a resistivity of $1.097 \Omega \cdot \mathrm{m}$ at $55^{\circ} \mathrm{C}$. Permeability and porosity were measured by AP608 (produced by the Coretest company). The relative permeability was measured by unsteadystate method (temperature: $55^{\circ} \mathrm{C}$, pore pressure: $14 \mathrm{Mpa}$, and pressure of surrounding rock: $30 \mathrm{Mpa}$ ). Figure 1 shows the nuclear magnetic $T_{2}$ distribution of each sample measured under the saturated water conditions. Figure 2 presents the relationship between the resistivity index and the water saturation. 


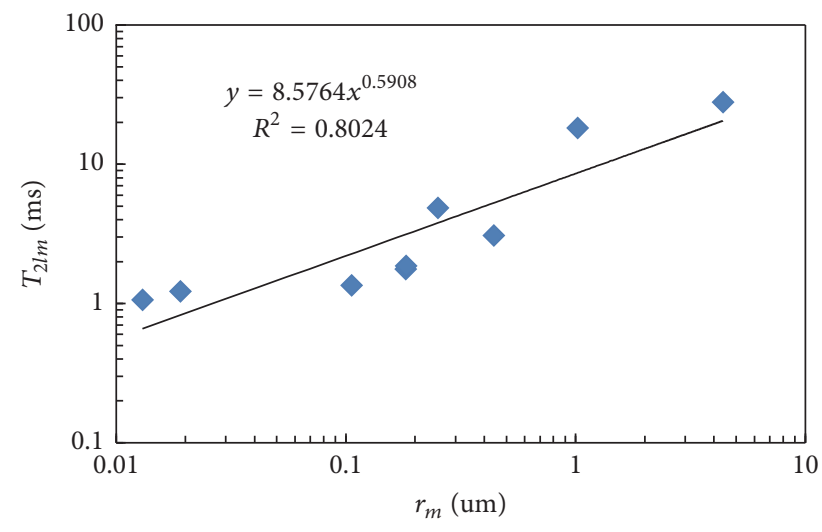

FIGURE 3: Relationship between $T_{2 l m}$ and $r_{m}$ in the study area.

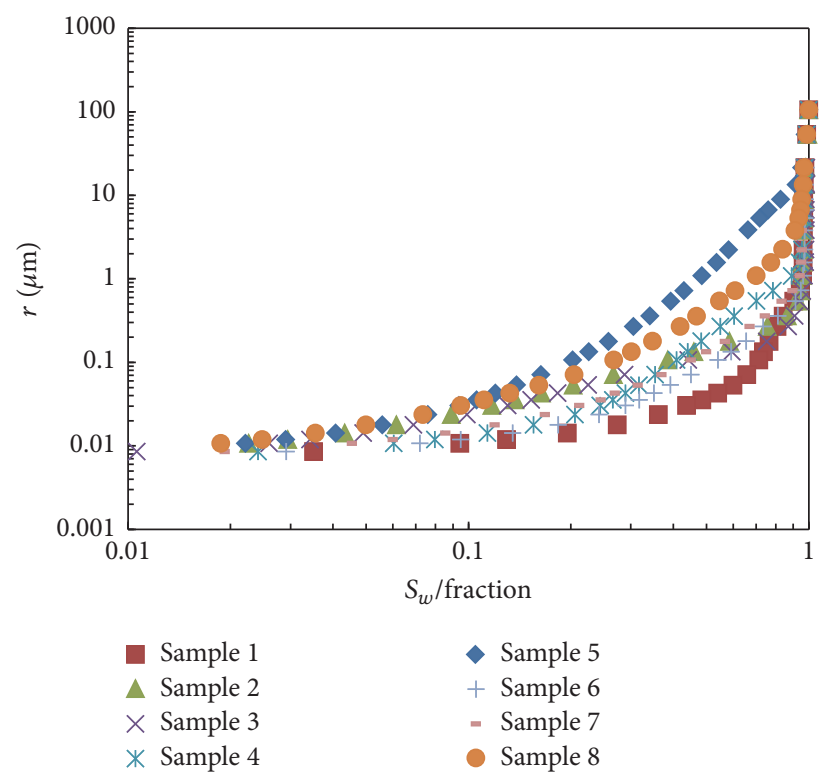

FIGURE 4: Relationship between the pore radius and wetting phase saturation transformed on the basis of a capillary pressure curve.

Figure 3 is the relationship between the $T_{2}$ logarithmic average $\left(T_{2 l m}\right)$ and average pore throat radius $\left(r_{m}\right)$ in the study area within the sample selection. The average pore throat radius $\left(r_{m}\right)$ can be obtained from mercury injection data. According to (6), $m_{t}=8.5764$ and $n_{t}=0.5908$ can be obtained.

As viewed from Figure 4, in combination with (15), a multifractal dimension phenomenon existed in this region. Through analysis of the data, the mutation position of the fractal dimension change was identified as the piece-wise point. According to the pore radius of $0.03 \mu \mathrm{m}$ and $1 \mu \mathrm{m}$ as the dividing point, the pores were divided into small, medium, and large categories. Equation (4) of the piece-wise method was applied for the transformation between the resistivity index and the capillary pressure, and the transformation results are as shown in Figure 5. The adoption of the piecewise method was significantly more effective than the results of the model of Li [9], which indicated that it was more

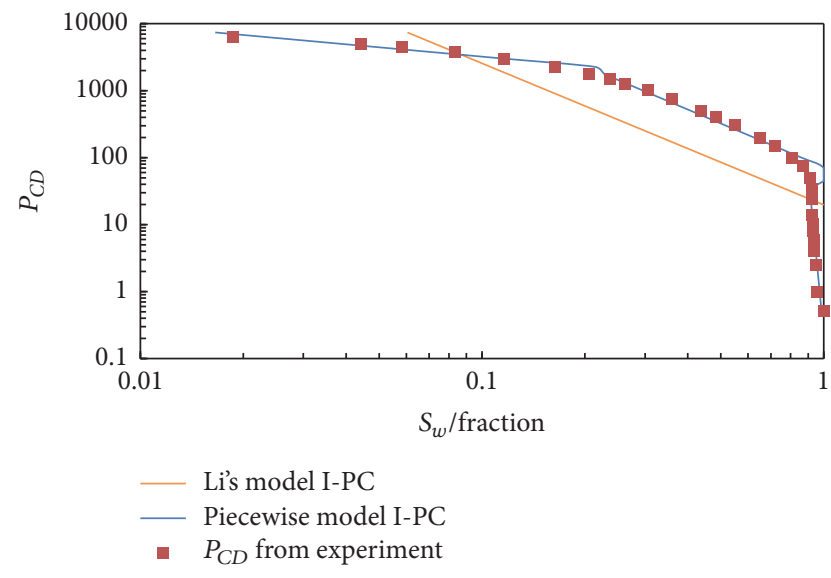

Figure 5: $I-P_{C}$ transformed by the piece-wise method.

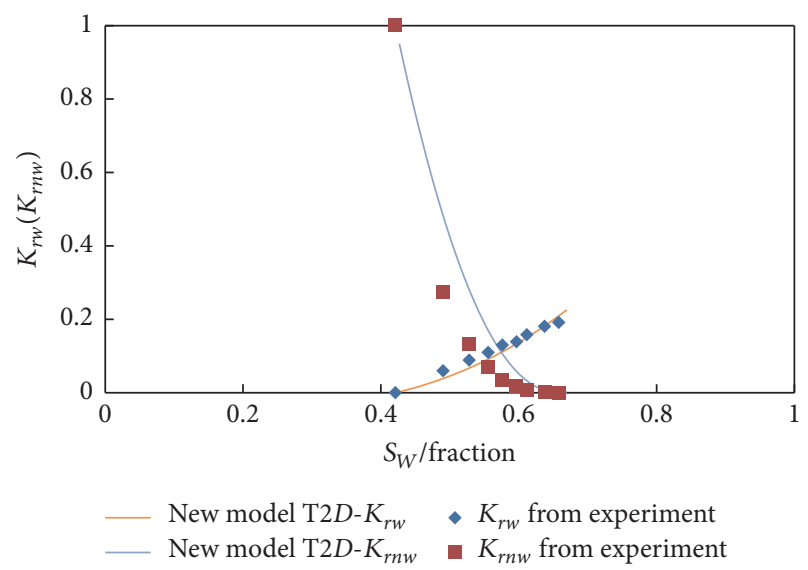

FIGURE 6: Transformation result contrast of Sample 4.

reasonable to use the described piece-wise transformation method in this region.

Table 1 lists the basic information of the cores which were taken from a tight sandstone reservoir in northwest China, including porosity, permeability, rock-electro parameters $b$ and $n$, and the piece-wise fractal dimension of the $T_{2}$ distribution. In the table, the last three columns were obtained on the basis of the piece-wise point in combination with (17). The lower $D_{f}$ is found to be, the higher the pore frequency is. Therefore, the cores were clearly mainly composed of small pores, with fewer large pores present.

Figure 6 is the contrast diagram of the relative permeability curve of Sample 4, which was obtained through laboratory measurements and which was transformed by $T_{2 D}$. It is worth nothing that the transformation parameter was solved by the previously determined piece-wise method. And the judgment coefficient of the wetting phase's relative permeability was 0.934 . The nonwetting phase's relative permeability was calculated by (21). Results indicate that there is a certain error between the calculated value and actual value. Figure 7 shows the transformation results of Sample 5; the judgment coefficient of the wetting phase's relative permeability was 0.875. After analyzing the sample data in this region, (21) 
TABLE 1: Basic information of the rock samples.

\begin{tabular}{lccccccc}
\hline Core number & $\Phi(\%)$ & $K(\mathrm{md})$ & $n$ & $b$ & $D_{f T 2}$ (large) & $D_{f T 2}(\mathrm{medium})$ & $D_{f T 2}(\mathrm{small})$ \\
\hline 1 & 6.487 & 0.019 & 2.19 & 0.9924 & 2.9875 & 2.8631 & 1.7245 \\
2 & 9.646 & 0.11 & 1.191 & 1.0003 & 2.9123 & 2.6573 & 1.6317 \\
3 & 11.791 & 0.097 & 1.25 & 0.9943 & 2.9375 & 2.6445 & 1.2662 \\
4 & 12.286 & 9.402 & 1.552 & 1.0210 & 2.8862 & 2.7258 & 1.2623 \\
5 & 10.542 & 15.313 & 1.541 & 0.9963 & 2.8944 & 2.6689 & 1.2610 \\
6 & 8.588 & 0.036 & 1.223 & 1.0169 & 2.9850 & 2.8330 & 1.6979 \\
7 & 4.507 & 0.041 & 2.143 & 1.0245 & 2.9721 & 2.7554 & 1.6070 \\
8 & 10.223 & 0.101 & 1.353 & 1.0097 & 2.9425 & 2.8911 & 1.7435 \\
\hline
\end{tabular}

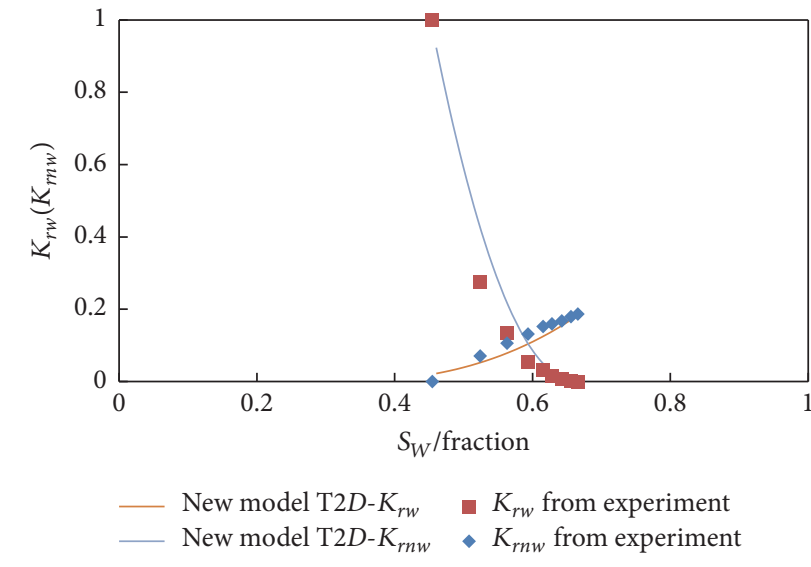

FIGURE 7: Transformation result contrast of Sample 5.

was improved upon when calculating the nonwetting phase's relative permeability.

$$
\begin{aligned}
K_{r n w} & =\left(1-S_{w}^{*}\right)^{2}\left(1-\left(S_{w}^{*}\right)^{\lambda /(2+\lambda)}\right)\left(\frac{1-S_{w}-S_{o r}}{1-S_{o r}-S_{i r}}\right) \\
& =\left(1-S_{w}^{*}\right)^{3}\left(1-\left(S_{w}^{*}\right)^{\lambda /(2+\lambda)}\right) .
\end{aligned}
$$

Figures 8 and 9 show the nonwetting phase's relative permeability for Samples 4 and 5, which were calculated by (24), respectively. Table 2 lists the fitting coefficient $R^{2}$, as well as the error contrast of the two types of calculation methods. The calculation results of (24) were determined to be more accurate than that of (22). A similar factor was added by Ma et al. [15] to the model of Li [9] in the calculation of $k_{r w}$, in order to introduce the pore tortuosity. Equation (24) illustrated the improvement in $K_{r n w}$ in accordance with $k_{r w}$ model presented by $\mathrm{Ma}$ et al. [15]. However, the introduction of the factor and the relationship between the effective nonwetting phase flow path and the pore tortuosity require further research.

It is worth noting that we get the pseudo relative permeability curve by $T_{2}-K_{r}$ model, the same as Yokoyama and Lake [16] and Slijkerman et al. [17] get pseudo capillary pressure curve by $T_{2}-P_{C}$. This is just a kind of approximate method

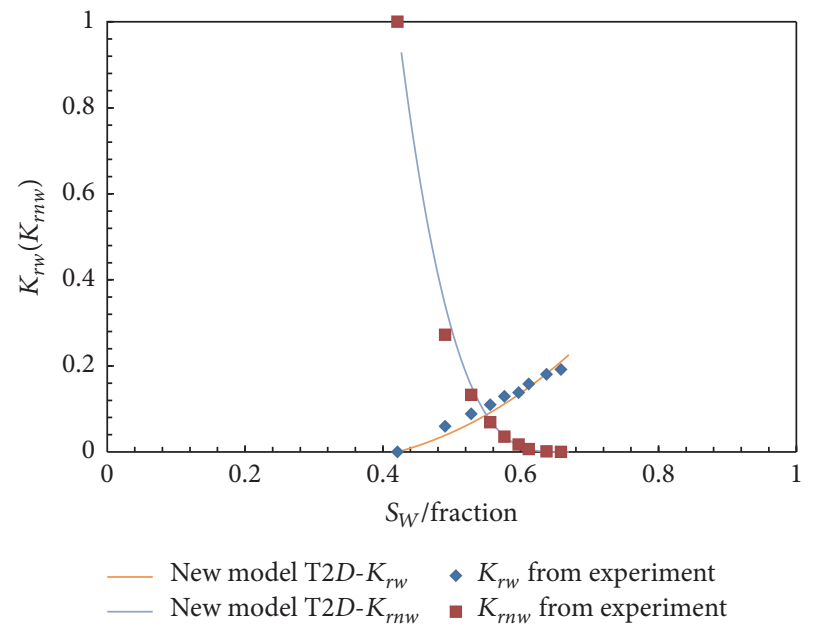

FIgURE 8: Nonwetting phase's relative permeability of Sample 4 calculated by (24).

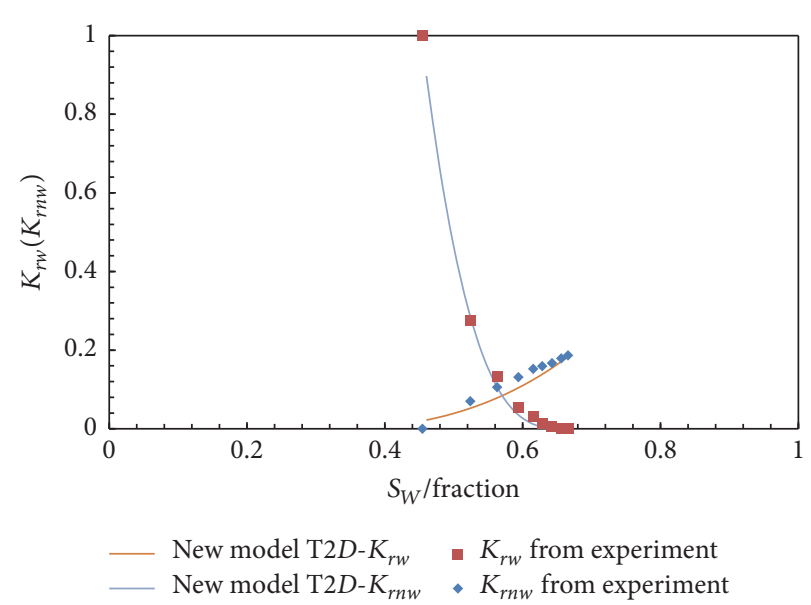

FIGURE 9: Nonwetting phase's relative permeability of Sample 5 calculated by (24).

to obtain relative permeability. In the case of the price of crude oil being low, this method can save time and reduce the cost. At the same time we also can anticipate $T_{2}-K_{r}$ model application prospect in the actual logging. 
TABLE 2: Calculation result contrast of the different equations.

\begin{tabular}{lcccc}
\hline \multirow{2}{*}{ Core number } & \multicolumn{2}{c}{ Equation (24) } & \multicolumn{2}{c}{ Equation (21) } \\
& Mean absolute error & $R^{2}$ & Mean absolute error & $R^{2}$ \\
\hline 4 & 1.4488 & 0.9939 & 6.6979 & 0.8983 \\
5 & 1.0624 & 0.9975 & 3.7613 & 0.9602 \\
\hline
\end{tabular}

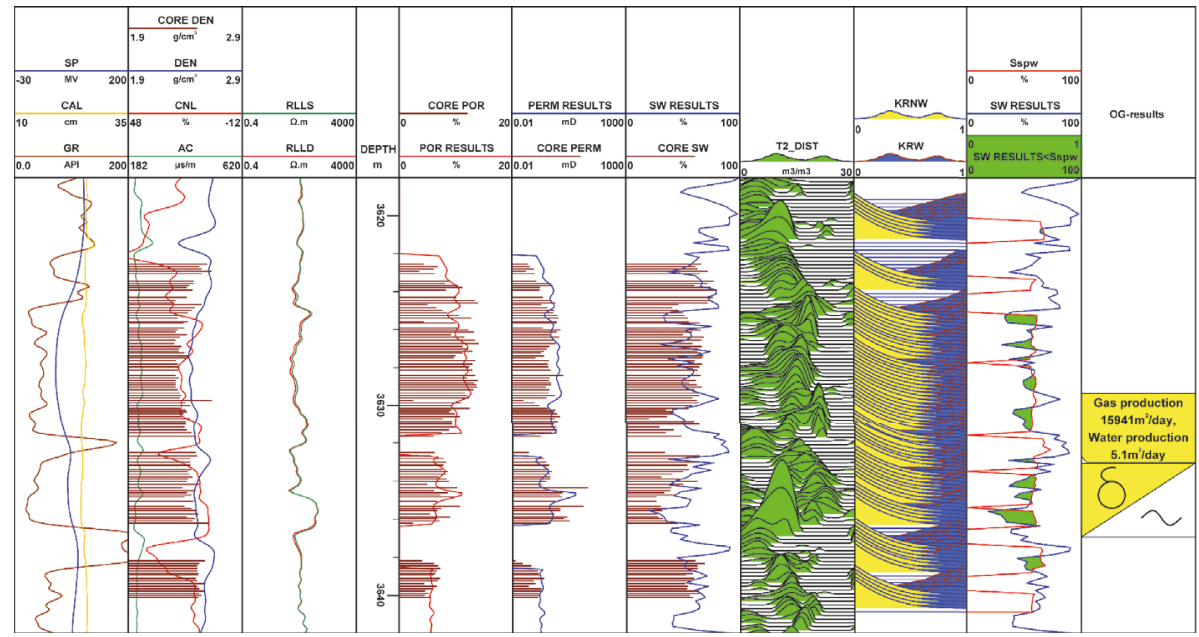

Figure 10: Comprehensive logging interpretation chart of Well M.

\section{Application Case Analysis}

The transformation model of the relative permeability and transverse relaxation time is obtained and verified, based on the processing and research of the experimental data, and according to the regional characteristics, the piece-wise method is adopted. This model is applied for the processing of the nuclear magnetic logging data, in order to obtain the relative permeability curve with changes over depth. It must be pointed out that the results we get by this method are pseudo relative permeability curves, as well as using $T_{2}$ spectrum to calculate pseudo capillary pressure curve which is widely used now. By simulating the underground conditions (temperature, pressure, the instrument measuring frequency, and fluid properties), we can obtain the results which were close to the real underground logging response value. Temperature impacts magnet magnetic field intensity; it has great influence on the result of the nuclear magnetic measurement. However, pressure mainly affects the structure of the rock itself. It should be pointed out that, due to the limitation of equipment, we cannot do nuclear magnetic experiment under the condition of high pressure. But the cores are measured after being saturated with water under high pressure, and the measured time is short. We hold the view that such a short time for the influence of the pore structure is negligible. At the same temperature and measuring frequency, NMR lab measurements results can be close to the actual logging data. We proposed a new method to identify the reservoir accurately based on $T_{2}-K_{r}$ model.
The processing was carried out on the target segment of Well $\mathrm{M}$ in the tight sandstone of the study area. Nuclear magnetic logging data were used to obtain the relative permeability curve with changes over depth. Figure 10 shows the comprehensive logging interpretation chart of Well $M$, in which Tracks 4-6 list the porosity, permeability, water saturation, and core data obtained by logging. Track 7 is $T_{2}$ distribution from NMR logging. Track 8 is the relative permeability curve calculated by the $T_{2}-K_{r}$ model. It should be noted that the relative permeability curve could not be obtained at all of the depth points, and the mudstone layer influenced the transformation. In track 9, the $S s p w$ is the saturation corresponding to the wetting and nonwetting phase permeability equal points, and SW RESULTS is the standardized wetting phase water saturation. The cases of the $S W$ RESULTS greater than the Sspw indicate that this layer produced much water (per unit volume). In contrast, the cases of the $S w^{*}$ less than the Sspw indicate that this layer produced much gas (per unit volume), which is consistent with the gas testing results (in track 10). It is worth nothing that green overlapping in track 9 has shown the case of producing much gas $\left(S W\right.$ RESULTS $\left.<S_{s p w}\right)$. At the depth ranges from 3632.7 to $3636.6 \mathrm{~m}$, there is a gas-water layer of which gas production is $15941 \mathrm{~m}^{3} /$ day and water production is $5.1 \mathrm{~m}^{3} /$ day. It has been proven that this method will be of a guiding significance for the exploration of oil and gas layers in hydrocarbon developments. We provide a very intuitive method to monitor reservoir production. 


\section{Conclusions}

In this study, a transformation model between the transverse relaxation time $T_{2}$ and the relative permeability $K_{r}$ was established through the analysis of the physical rock experimental data. In the model, the transformation parameters were solved using the transformation relationship between the physical variables, such as the capillary pressure, transverse relaxation time, and resistivity index. In the processing of the experimental data, continuous fractal dimension phenomena were found to exist in the samples from the study area. Therefore, a piece-wise method was used to calculate the parameters. The piece-wise points were determined according to the mutation position of the fractal dimension, and the pore radiuses of $0.03 \mu \mathrm{m}$ and $1 \mu \mathrm{m}$ were selected as the dividing points. During the application of the Li model, the capillary pressure curve obtained by the piece-wise transformation of the resistivity index was shown to be relatively consistent with that obtained by the experiments, thereby proving the rationality of the piece-wise method. During the use of the piece-wise method and the new model, the wetting phase's relative permeability transformed by the transverse relaxation time was shown to be relatively consistent with that obtained by the experimental measurements. However, the nonwetting phase's relative permeability obtained by the improved model had better effects, which confirmed that this model was reasonable and effective. The model was applied to the evaluation of the reservoir logging. Meanwhile, the nuclear magnetic logging data were used in this study to obtain the relative permeability curve with depth and for identifying major water and gas production horizons, which were consistent with the gas testing results. It was indicated that this model had practical application significance. Our new method to identify the reservoir is effective and feasible.

It was also determined that a specific problem existed in this model. The $T_{2}$ distribution measured under the wetting phase saturation state was applied for the calculation of the transformation parameters in this study. However, the oil and gas had in fact an impact on the $T_{2}$ distribution, which was ignored in this study. The focus of future research should be to correct this impact through measuring the transverse relaxation time and relative permeability under different saturation states, in order to improve the model.

\section{Conflicts of Interest}

The authors declare that they have no conflicts of interest.

\section{Acknowledgments}

This work is supported in part by the National Natural Science Foundation of China under Grant 41174096 and the Graduate Innovation Fund of Jilin University under Project 2016103.

\section{References}

[1] L. Z. Xiao, Z. B. Qiang, W. S. Wu et al., Frontiers Investigation in Well Logging, vol. II, Petroleum Industry Press, 2007.
[2] W. R. Purcell, "Capillary pressures-their measurement using mercury and the calculation of permeability therefrom," Journal of Petroleum Technology, vol. 1, no. 2, p. 39, 1949.

[3] R. H. Brooks and A. T. Corey, "Properties of porous media affecting fluid flow," Journal of the Irrigation and Drainage Division, vol. 6, p. 61, 1966.

[4] C. M. Zhang, Z. B. Chen, Z. S. Zhang et al., "Fractal characteristics of reservoir rock pore structure based on $\mathrm{NMR} \mathrm{T}_{2}$ distribution," Journal of Oil and Gas Technology, vol. 29, no. 4, pp. 80-86, 2007.

[5] P. Pfeifer and D. Avnir, "Chemistry in no integral dimensions between two and three: I. Fractal theory of heterogeneous surfaces," Journal of Chemical Physics, vol. 79, pp. 3369-3558, 1983.

[6] X. M. Ge, Y. R. Fan, F. Wu et al., "Correspondence of core nuclear magnetic resonance $\mathrm{T}_{2}$ spectrum and resistivity index," Journal of China University of Petroleum, vol. 36, no. 6, pp. 5356, 2012.

[7] S.-T. Bai, J.-B. Wan, D.-J. Cheng et al., "Study of relationship between petrophysical experimental parameters: capillary pressure, NMR $\tau_{2}$ distribution, resistivity index, relative permeability," Journal of Chengdu University of Technology (Science \& Technology Edition), vol. 41, no. 4, pp. 483-491, 2014.

[8] P. G. Toledo, R. A. Novy, H. T. Davis, and L. E. Scriven, "Capillary pressure, water relative permeability, electrical conductivity and capillary dispersion coefficient of fractal porous media at low wetting phase saturations," SPE Advanced Technology Series, vol. 2, no. 1, pp. 136-141, 1994.

[9] K. Li, "Calculation of gas-water relative permeability from resistivity and the comparison with experimental data," GRC Transactions, vol. 30, pp. 825-830, 2006.

[10] Y. Volokitin and W. Looyestijn, "Constructing capillary pressure curves from NMR log data in the presence of hydrocarbons," in Proceedings of the SPWLA 40th Annual Logging Symposium, Oslo, Norway, 1999.

[11] Y. D. He, Z. Q. Mao, L. Z. Xiao et al., "A new method to obtain capillary pressure curve using $\mathrm{NMR}_{2}$ distribution," Journal of Jilin University (Earth Science Edition), vol. 35, no. 2, pp. 177-181, 2005.

[12] Y.-D. He, Z.-Q. Mao, L.-Z. Xiao, and X.-J. Ren, "An improved method of using NMR $\mathrm{T}_{2}$ distribution to evaluate pore size distribution," Chinese Journal of Geophysics, vol. 48, no. 2, pp. 373-378, 2005.

[13] K. Li and R. N. Horne, "Comparison of methods to calculate relative permeability from capillary pressure in con-solidated water-wet porous media," Water Resources Research, vol. 42, no. 6, Article ID W06405, 2006.

[14] K. Li, "A new method for calculating two-phase relative permeability from resistivity data in porous media," Transport in Porous Media, vol. 74, no. 1, pp. 21-33, 2008.

[15] D. Ma, C. Liu, and C. Cheng, "New relationship between resistivity index and relative permeability," Journal of Energy Resources Technology, vol. 137, no. 3, Article ID 032904, 2015.

[16] Y. Yokoyama and L. W. Lake, "The effects of capillary pressure on immiscible displacements in stratified porous media," in Proceedings of the SPE Annual Technical Conference and Exhibition, Society of Petroleum Engineers, 1981.

[17] W. F. J. Slijkerman, J. P. Hofman, W. J. Looyestijn, and Y. Volokitin, "A practical approach to obtain primary drainage capillary pressure curves from NMR core and log data," Petrophysics, vol. 42, no. 4, 2001 

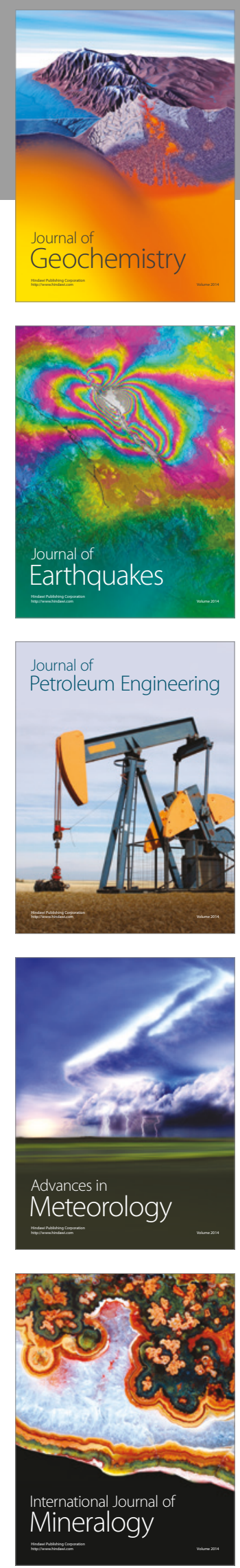
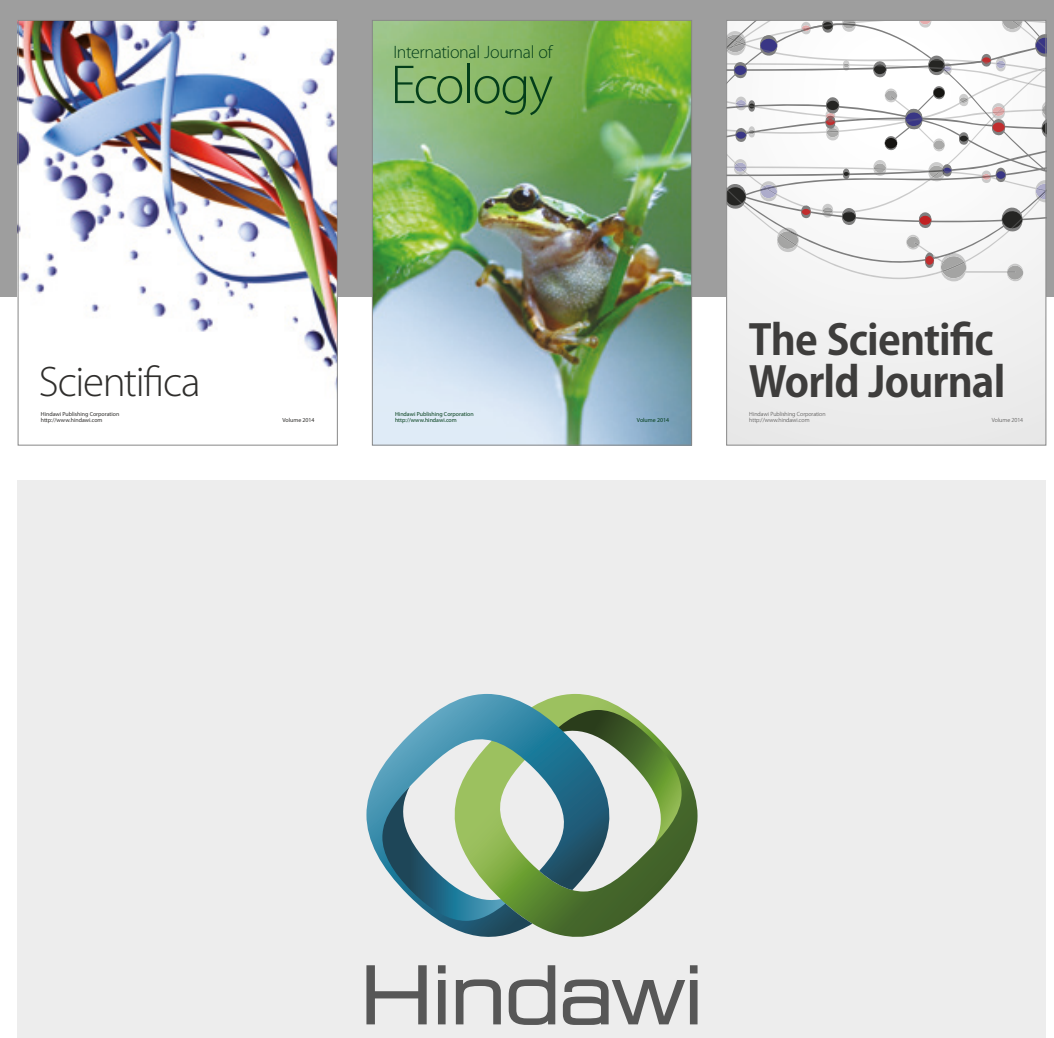

Submit your manuscripts at

https://www.hindawi.com
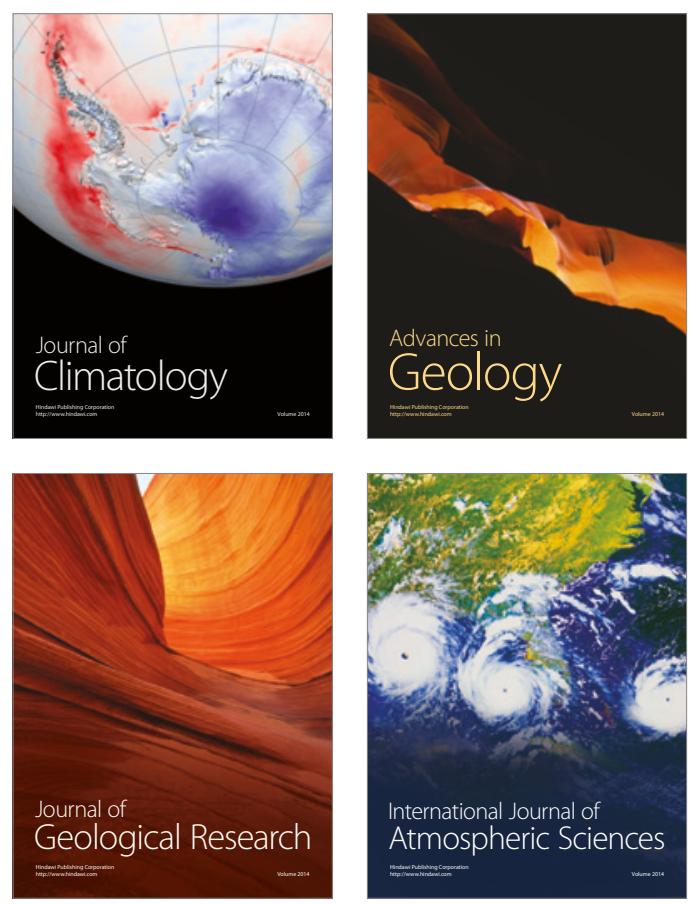

The Scientific

World Journal
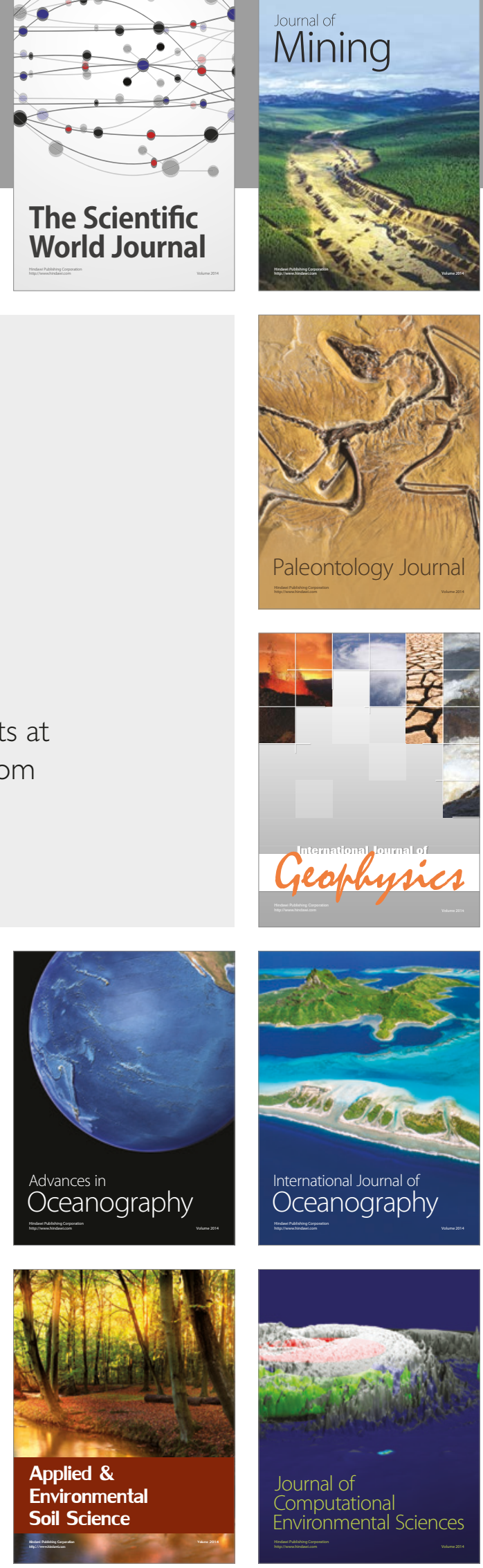